\title{
Danno renale acuto da mezzi iodati di contrasto. Fisiopatologia e suggerimenti di prevenzione
}

\author{
Michele Andreucci \\ Professore Associato dell'Università "Magna Graecia" di Catanzaro, Cattedra di Nefrologia, Dipartimento di "Scienze della Salute", \\ Campus "Salvatore Venuta", Università "Magna Graecia”, Catanzaro
}

\begin{abstract}
lodinated contrast-induced acute kidney injury. Pathophysiology and prevention
The term acute renal failure (ARF) secondary to ischemic/nephrotoxic injury should no longer be used and be replaced by acute kidney injury (AKI), indicating an abrupt (within 24-48 hours) increase in serum creatinine by $0.5 \mathrm{mg} / \mathrm{dL}$ or by $25 \%$ above baseline. Replacing "failure" with "injury" served to include the entire range of renal impairment from small changes in serum creatinine to complete loss of renal function. The term ARF should be limited to a decrease in renal function so severe as to require dialysis. When AKI is due to radiographic contrast agents, it is called contrast-induced (CI) AKI. The pathogenesis of $\mathrm{Cl}-\mathrm{AKI}$ is complex and not well known. Many factors are involved, including hemodynamic changes such as renal vasoconstriction, particularly in the outer medulla where tubular segments devoted to active sodium reabsorption are located; the vasoconstriction may be due to direct action of the contrast agent but may also be mediated by stimulation of angiotensin II and adenosine, or by a decrease in the local production of prostaglandins and nitric oxide, all leading to medullary hypoxia. Other factors are osmotic diuresis (with increased tubular reabsorption of sodium and tubular epithelium injury, both worsening medullary hypoxia) and production of reactive oxygen species that will cause endothelial and epithelial damage, likewise worsening medullary hypoxia. The fall in renal blood flow and tubular obstruction will decrease the glomerular filtration rate. Treatment and prevention of $\mathrm{Cl}-\mathrm{AKI}$ are based on opposing these factors by adequate hydration; administration of furosemide along with fluid replacement; theophylline and aminophylline; $\mathrm{PGE}_{1}$ and $\mathrm{PGI}_{2} ; \mathrm{N}$-acetylcysteine, ascorbic acid and vitamin $\mathrm{E}$; nebivolol and statins.
\end{abstract}

Keywords: Acute renal failure, Acute kidney injury, AKI, Contrast-induced AKI, lodinated radiographic contrast agents, Pathogenesis of contrast nephrotoxicity

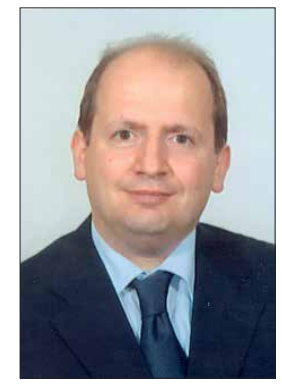

II termine Insufficienza Renale Acuta (IRA; per gli anglosassoni ARF, acronimo di Acute Renal Failure), dovuta a cause tossico-ischemiche, è stato da alcuni anni sostituito dal termine Acute Kidney Injury (AKI) (1) che tradurrei in italiano "Danno Renale Acuto". Con questo termine si indica un'improvvisa e rapida (entro 24-48 ore) diminuzione del Filtrato Glomerulare (GFR) che causa una ritenzione di liquidi e

Michele Andreucci

Accepted: November 29, 2016

Published online: January 20, 2017

Indirizzo per la corrispondenza:

Michele Andreucci, MD, PhD

Professore Associato di Nefrologia

Università della "Magna Graecia"

Viale Europa, loc. Germaneto

88100 Catanzaro

andreucci@unicz.it di cataboliti e alterazioni del bilancio elettrolitico e acido-base $(2,3)$. Include un ampio range di compromissione renale che va da modesti aumenti della Creatinina sierica ( $\mathrm{CrS}$ ) fino alla perdita quasi completa della funzione renale (3). II termine IRA (ARF) è invece limitato alla perdita completa della funzione renale tale da richiedere la terapia dialitica (4).

Il danno renale acuto (AKI) può essere dovuto all'uso di mezzi di contrasto (MC) iodati a scopo diagnostico. È la $\mathrm{Ne}$ fropatia da MC (CIN, Contrast-Induced Nephropathy), una riduzione della funzione renale che si verifica da 24 a 72 ore dopo l'iniezione intravascolare di un mezzo di contrasto che non può essere spiegata da altre cause. Di solito non oligurica e asintomatica, è diagnosticata da un aumento della $\mathrm{CrS}$ (di $0.5 \mathrm{mg} / \mathrm{dL}$ o più, oppure da un aumento del $25 \%$ o più sulla $\mathrm{CrS}$ di base) che raggiunge il massimo valore tra il $3^{\circ}-5^{\circ}$ giorno per poi tornare ai valori di base entro $10-14$ giorni (5-7). In alcuni casi è una IRA con oliguria ( $400 \mathrm{~mL} / 24$ ore) che richiede la terapia dialitica; in questi casi la mortalità è elevata.

Le caratteristiche cliniche e il trattamento della Nefropatia da MC sono gli stessi delle AKI tossico-ischemiche da altre cause. 


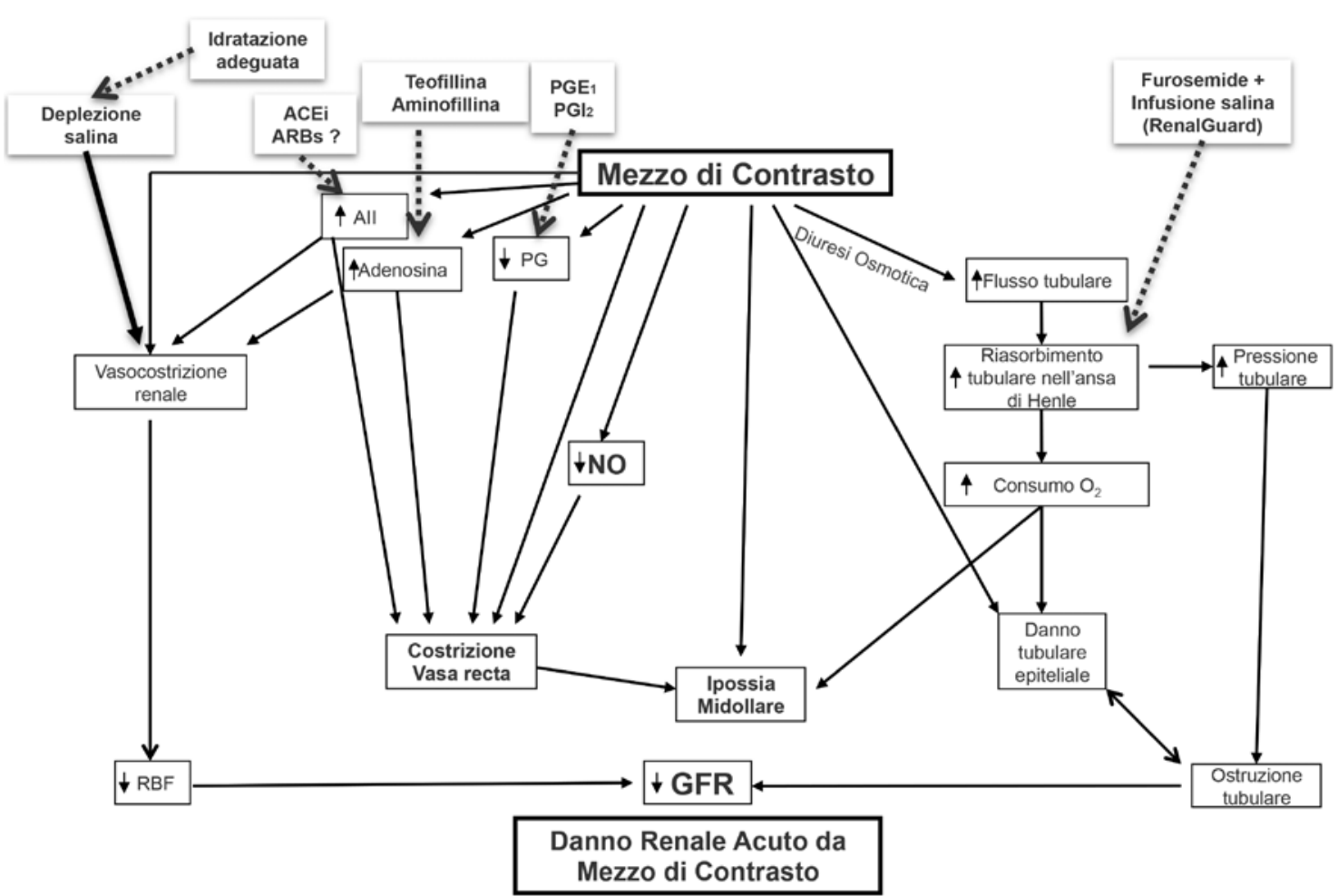

Fig. 1 - Patogenesi della Nefropatia da MC. Meccanismi emodinamici. (La freccia spessa indica stimolo. La freccia tratteggiata indica inibizione). $\mathrm{NO}=$ ossido nitrico

\section{Patogenesi}

La patogenesi della Nefropatia da MC non è completamente conosciuta anche se molti fattori sono stati dimostrati svolgervi un ruolo più o meno importante.

\section{Meccanismi emodinamici}

Molti studi sperimentali e clinici hanno dimostrato che I'iniezione intravascolare di un mezzo di contrasto causa una immediata vasodilatazione renale con aumento del flusso ematico renale (RBF, Renal Blood Flow). Questa è seguita da una prolungata vasocostrizione con aumento delle resistenze vascolari intrarenali e conseguente riduzione del RBF che di per sé riduce il GFR (Fig. 1).

L'effetto vasocostrittivo dei MC sui vasa recta è stato dimostrato da Sendenski et al (8) in esperimenti in vitro. Gli autori hanno isolato segmenti di vasa recta discendenti della midollare esterna di reni di ratto e li hanno microperfusi con una soluzione buffer contenente iodixanolo, un mezzo di contrasto non-ionico isosmolare (osmolalità di circa $290 \mathrm{mOsm} / \mathrm{kg}$ ), con concentrazione di lodio $23 \mathrm{mg} / \mathrm{mL}$, come negli esami radiologici nell'uomo. I risultati hanno dimostrato che lo iodixanolo: a) ha un effetto vasocostrittivo diretto (riduzione del lume vascolare del 52\%) sui vasa recta; b) aumenta la vasocostrizione da Angiotensina II; e c) riduce notevolmente (dell'82\%) la biodisponibilità dell'Ossido Nitrico (NO).

Anche l'Adenosina sarebbe coinvolta nella vasocostrizione da mezzo di contrasto. Infatti Arakawa et al (9) hanno dimostrato che in cani con ridotta funzione renale l'iniezione del mezzo di contrasto ioexolo induceva l'attivazione dei recettori dell' Adenosina $A_{2}$, che causava una iniziale vasodilatazione renale, e dei recettori dell'Adenosina $A_{1}$, che causava invece una prolungata vasocostrizione con alterazione dell'emodinamica renale; questa veniva prevenuta da antagonisti dell'Adenosina.

La vasocostrizione renale induce un'ischemia del rene che però non è uniforme, ma è più accentuata nella midollare renale esterna (10). Per comprendere questa situazione fisiologica dobbiamo esaminare la vascolarizzazione midollare.

II riassorbimento attivo del sodio (che richiede ossigenazione) da parte dei tubuli renali si verifica nel segmento S3 dei tubuli contorti prossimali e nel lembo spesso ascendente midollare delle anse di Henle che sono allocati nella midollare renale esterna, un ampio tessuto renale irrorato dai vasa recta. Questi però decorrono a una notevole distanza dalla maggior parte della midollare renale esterna, rendendo così difficile la sua ossigenazione. Pertanto, in condizioni fisiologiche, l'apporto di $\mathrm{O}_{2}$ alla midollare renale esterna è modesto con un conseguente scarso apporto di $\mathrm{O}_{2}$ ai segmenti tubulari renali, sede del riassorbimento attivo del sodio. Le Prostaglandine prodotte nella midollare renale, I'Ossido Nitrico (NO) e l'Adenosina controllano questa situazione, adeguando l'attività di trasporto attivo tubulare renale al limitato apporto di ossigeno, sia diminuendo il trasporto attivo tubulare sia aumentando il flusso ematico regionale (11). Qualsiasi (anche modesto) difetto in questi complessi meccanismi di controllo 
causa un'ipossia midollare (ischemia da discrepanza, carenza di $\mathrm{O}_{2}$ di fronte a un'aumentata richiesta di ossigenazione).

Se iniettiamo e.v. o i.a. un mezzo di contrasto iodato in un individuo normale con queste condizioni anatomo-funzionali, la vasocostrizione che ne consegue renderà particolarmente grave l'ipossia midollare renale (Fig. 1).

Vari fattori possono aggravare l’ipossia midollare renale:

1) la deplezione idrosalina del paziente che causa una vasocostrizione generalizzata aggravando la vasocostrizione renale da mezzo di contrasto;

2) la diuresi osmotica indotta dal mezzo di contrasto accentua l'ischemia da discrepanza perché aumenta l'afflusso di fluido tubulare ai segmenti tubulari di riassorbimento attivo di sodio; il maggiore apporto di sodio aumenterà il suo riassorbimento attivo con una conseguente maggiore richiesta di $\mathrm{O}_{2}$ che accentua l'ipossia midollare renale.

L'elevato fisiologico riassorbimento dell'ultrafiltrato glomerulare nei tubuli renali prossimali aumenta la concentrazione del mezzo di contrasto nel lume tubulare. Ne consegue un progressivo aumento dell'osmolalità del fluido tubulare; per il rapporto esponenziale concentrazione-viscosità, si avrà uno sproporzionato aumento della viscosità di questo fluido tubulare $(10,12,13)$. Siccome il flusso di un fluido in un tubo aumenta con il gradiente di pressione e diminuisce con la resistenza al flusso e siccome la resistenza aumenta proporzionalmente alla viscosità del fluido, l'aumentata viscosità del fluido tubulare causato dal mezzo di contrasto aumenterà la pressione endotubulare (13). Si crea così una condizione di ostruzione tubulare che contribuisce sia al danno dell'epitelio tubulare renale sia alla diminuzione del GFR (Fig. 1) (10).

\section{Note di Prevenzione}

\section{Adeguata idratazione}

Sulla base di quanto detto, il più importante procedimento per prevenire la vasocostrizione del mezzo di contrasto e presumibilmente la Nefropatia da mezzo di contrasto è un'adeguata idratazione del paziente prima di sottoporlo a infusione di mezzo di contrasto. La vecchia consuetudine di far limitare al paziente, che deve sottoporsi a un esame radiologico con mezzo di contrasto, l'ingestione di liquidi dal giorno precedente è stata ormai da tempo abbandonata. Anzi, essa deve essere sostituita dal suggerimento di assunzione di supplementi di acqua da parte del paziente per poter così ottenere una diuresi di almeno $1 \mathrm{~mL} / \mathrm{min}$. Anzi, più che acqua per os è necessario somministrargli e.v. una soluzione salina $(0.9 \% \mathrm{NaCl}, 1 \mathrm{~mL} / \mathrm{kg} / \mathrm{ora})$, da iniziare 6 -12 ore prima e continuare sino a 12-24 ore dopo l'esame radiografico (Fig. 1). L'infusione salina, espandendo il volume extracellulare del paziente, da una parte riduce il riassorbimento idrosodico attivo nei segmenti prossimali dei tubuli renali riducendo il consumo di ossigeno e quindi l'ischemia da discrepanza, dall'altra invece aumenta la velocità di flusso del fluido endotubulare riducendo conseguentemente il tempo di contatto dell'epitelio tubulare renale con il mezzo di contrasto e quindi la sua azione nefrotossica diretta (anche per la maggiore diluizione del mezzo di contrasto nel lume dei tubuli renali). Inoltre l'espansione del volume intravascolare sopprime il sistema Renina-Angiotensina con conseguente riduzione della vasocostrizione renale e dell'ipoperfusione del rene (quindi con un conseguente miglioramento del RBF).

Studi clinici e di meta-analisi hanno dimostrato che l'infusione di Bicarbonato di sodio (per esempio, soluzione di sodio bicarbonato $154 \mathrm{mEq} / \mathrm{L}, 3 \mathrm{~mL} / \mathrm{kg} /$ ora un'ora prima, seguito da $1 \mathrm{~mL} / \mathrm{kg}$ /ora per 6 ore durante e dopo l'esame radiografico) è preferibile all'infusione di soluzione di $\mathrm{NaCl}$ (14-17), perché agli effetti positivi sopra descritti si aggiunge una minore acidificazione delle urine e della midollare renale che così riduce la produzione e aumenta la neutralizzazione dei radicali liberi di ossigeno, che svolgono un importante ruolo nella nefrotossicità dei MC (17-20) (vedi oltre). Vi sono però autori che hanno negato una maggiore efficacia preventiva del Bicarbonato di sodio rispetto alla soluzione salina (21-24). Vi è persino chi ha trovato un aumento dell'incidenza della Nefropatia da MC con l'uso e.v. del Bicarbonato di sodio (25). KDIGO (Kidney Disease Improving Global Outcomes Guidelines for Acute Kidney Injury Work Group) (26) "raccomanda l'espansione del volume extracellulare o con soluzione isotonica di cloruro di sodio o con soluzione di bicarbonato di sodio piuttosto che non espansione di volume nei pazienti a rischio di Nefropatia da mezzo di contrasto».

\section{Riduzione della ischemia da discrepanza}

La riduzione della ischemia da discrepanza si può ottenere o aumentando l'apporto di ossigeno alla midollare renale oppure riducendo il riassorbimento attivo di sodio.

Per aumentare l'apporto di ossigeno alla midollare renale è necessario correggere la vasocostrizione dei vasa recta.

Sulla base dei risultati sperimentali sopra descritti, si è pensato di ridurre la vasocostrizione renale usando la Teofillina o I'Aminofillina, in quanto antagonisti non selettivi dei recettori dell'Adenosina (Fig. 1); ma l'uso clinico nell'uomo della Teofillina o dell'Aminofillina ha dato risultati contrastanti: alcuni autori hanno infatti descritto effetti benefici nel prevenire la Nefropatia da MC $(27,28)$; altri autori invece no (29).

Abbiamo visto che i MC stimolano la produzione di Angiotensina II che contribuisce alla vasocostrizione renale. Questo ha fatto pensare all'utilità dell'utilizzo di ACE-inibitori (Angiotensin-Converting Enzyme inhibitors) o di ARBs (Angiotensin II Receptor Blockers) nel prevenire la Nefropatia da MC (Fig. 1). Invece si ritiene comunemente che questi farmaci possano avere persino degli effetti deleteri (30): così molti autori ritengono che sia gli ACE-inibitori che gli ARBs vadano sospesi nei pazienti con ridotta funzione renale prima di sottoporli a esami radiologici con mezzo di contrasto (31-33) 
soprattutto nei pazienti anziani (34). Altri autori invece negano che questi farmaci siano deleteri (35). Secondo KDIGO non ci sono sino ad oggi prove sufficienti a raccomandare la sospensione degli ACE-inibitori o degli ARBs prima della somministrazione del mezzo di contrasto nell'uomo (36).

Come si è detto in precedenza, nell'individuo normale le Prostaglandine, assieme all'Ossido Nitrico e all'Adenosina, controllano il mantenimento di un equilibrio tra l'attività di trasporto tubulare attivo e il limitato apporto di ossigeno nella midollare renale esterna, e il mezzo di contrasto aumenta la vasocostrizione da Angiotensina II e diminuisce i livelli di Prostaglandine. Questo ha fatto sperare che le Prostaglandine avessero un'efficacia protettiva contro gli effetti dei MC. Koch et al (37) e Sketch et al (38) hanno studiato in 117 pazienti con una CrS media di $2.2 \mathrm{mg} / \mathrm{dL}$ gli effetti protettivi verso i MC dell'infusione di Prostaglandina $\mathrm{PGE}_{1}$ per 6 ore (iniziando un'ora prima dell'infusione del mezzo di contrasto). Dopo 48 ore i pazienti che avevano ricevuto il placebo mostravano un aumento della $\mathrm{CrS}$ di $0.72 \mathrm{mg} / \mathrm{dL}$, mentre quelli che avevano ricevuto $20 \mathrm{ng} \mathrm{PGE}_{1} / \mathrm{kg} / \mathrm{min}$ presentavano un aumento della $\mathrm{CrS}$ solamente di $0.12 \mathrm{mg} / \mathrm{dL}$. Più interessante è il lavoro di Spargias et al $(39,40)$ che hanno condotto uno studio prospettico e randomizzato con lloprost $\left(\mathrm{PGI}_{2}\right.$, Prostaciclina). Gli autori hanno utilizzato una dose di Iloprost pari a $1 \mathrm{ng} / \mathrm{kg} / \mathrm{min}$, iniziando $30-90$ min prima sino a 240 min dopo la somministrazione del mezzo di contrasto iodato in pazienti sottoposti ad angiografia con intervento sulle coronarie. Si è così osservata una riduzione del rischio di Nefropatia da MC del 70\%.

Come si è già detto, l'aumentata attività di riassorbimento di sodio nel segmento S3 dei tubuli prossimali e nel lembo ascendente spesso delle anse di Henle causa un aumento del consumo di $\mathrm{O}_{2}$ contribuendo alla ipossia midollare (ischemia da discrepanza). Una riduzione di tale ischemia si potrebbe ottenere anche riducendo il riassorbimento attivo del sodio. Di qui l'uso della Furosemide che inibisce il riassorbimento attivo di sodio proprio in quei segmenti tubulari. Vari studi tuttavia hanno dimostrato nessuna protezione verso la $\mathrm{Ne}$ fropatia da MC, se non addirittura effetti deleteri, tanto da portare al consiglio di evitare l'uso dei diuretici prima dell'esposizione al mezzo di contrasto (41).

Si è visto in seguito che i risultati negativi con i diuretici erano dovuti all'ipovolemia conseguente alla perdita idrosalina con le urine. Questo avviene ancor più marcatamente negli anziani che di per sé tendono a perdere sale con le urine. Per evitare l'ipovolemia da diuretici è necessario infondere e.v. una quantità di fluidi che corrisponda esattamente al volume delle urine eliminato dal paziente sotto l'effetto della Furosemide (42). Questa procedura può essere ben realizzata mediante uno speciale apparecchio chiamato RenalGuard che consente il mantenimento della volemia mediante infusioni endovenose che eguagliano il volume di urine emesso sotto l'effetto della Furosemide; il bilancio viene così mantenuto automaticamente senza rischi per il paziente (Fig. 1) $(43,44)$.
Utilizzando il sistema RenalGuard, Briguori et al (43) hanno condotto uno studio multicentrico e randomizzato confrontando il ripristino delle perdite urinarie sotto Furosemide mediante infusione e.v. di soluzione salina senza (Gruppo Controllo) o con RenalGuard (Gruppo RenalGuard) in pazienti ad alto rischio sottoposti ad angiografia coronarica e/o periferica e/o angioplastica, con un eGFR $\leq 30 \mathrm{~mL} / \mathrm{min} / 1.73 \mathrm{~m}^{2}$ (estimated Glomerular Filtration Rate è la Clearance della Creatinine calcolata con una delle diverse formule). L'incidenza della Nefropatia da mezzo di contrasto risultò del 16\% nel Gruppo Controllo (idratazione standard) e del 5\% nel Gruppo RenalGuard, dimostrando così l'utilità del sistema RenalGuard. Risultati simili sono stati ottenuti da Marenzi et al (45) in pazienti con insufficienza renale cronica sottoposti a procedure coronariche; l'incidenza fu del $4.6 \%$ versus $18 \%$ del Gruppo Controllo $(p=0.005)$.

\section{II ruolo delle ROS (Reactive Oxygen Species) e dell'Ossido Nitrico}

L'ipossia midollare renale può causare la formazione delle specie reattive dell'ossigeno (ROS) (Fig. 2) (46). Le ROS possono causare danni diretti sulle cellule endoteliali e sull'epitelio tubulare e possono aggravare l'ipossia midollare (47).

Un ruolo importante è svolto dalla diminuzione del NO che si ritiene essere dovuta alla sua reazione con le ROS, in particolare con l'anione superossido $\mathrm{O}_{2}^{-}(48,49)$. Questa reazione può portare alla formazione del più potente anione ossidante perossinitrito $\left(\mathrm{ONOO}^{-}\right)$(50) che può essere ancora più dannoso per le cellule endoteliali (Fig. 2).

Anche in condizioni fisiologiche il trasporto attivo tubulare si associa alla formazione delle ROS, soprattutto nel lembo spesso ascendente dell'ansa di Henle (nella midollare renale esterna) dove la densa popolazione mitocondriale rappresenta un'importante sede di generazione di $\mathrm{O}_{2}^{-}(47,49)$. La somministrazione di un mezzo di contrasto aumenta la produzione delle ROS e dello stress ossidativo renale, che causa il danno delle membrane cellulari con conseguente apoptosi e necrosi soprattutto nel segmento S3 dei tubuli contorti prossimali e nel lembo spesso ascendente midollare delle anse di Henle (49).

Myers et al (51) hanno dimostrato, con esperimenti in vivo su ratti, che la riduzione del flusso ematico renale (corticale e midollare) causato dal mezzo di contrasto è in parte dovuto alla ridotta locale sintesi di NO. Pisani et al (52) hanno dimostrato che la manganese superossido dismutasi ricombinante, somministrata in vivo a ratti trattati con mezzo di contrasto, riduceva lo stress ossidativo e conseguentemente preveniva la riduzione del GFR e i danni istologici indotti dal mezzo di contrasto.

\section{Note di Prevenzione}

Considerata l'importanza dell'aumento delle ROS nella patogenesi della Nefropatia da $\mathrm{MC}$, si è pensato di utilizzare gli antiossidanti per prevenire questa Nefropatia. II primo e 


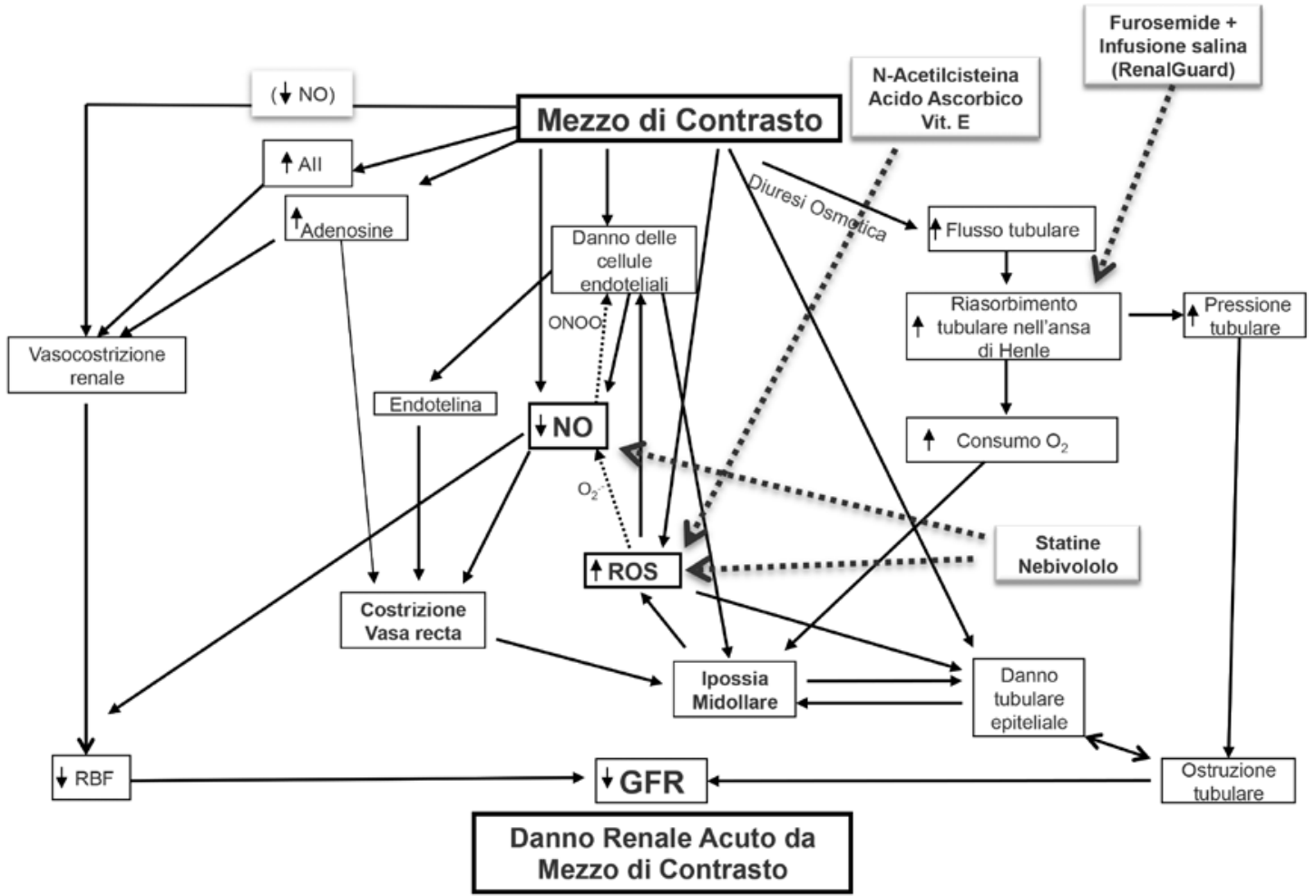

Fig. 2 - Patogenesi della Nefropatia da MC. II ruolo delle ROS (Reactive Oxygen Species) e dell'Ossido Nitrico (NO). (La freccia tratteggiata indica la reazione dei ROS con NO che causa una riduzione locale di NO e la formazione dell'anione perossinitrito, un potente ossidante che causa danno cellulare). più importante antiossidante usato è stata la N-Acetilcisteina (Fig. 2) $(53,54)$.

L'efficacia della N-Acetilcisteina è ancora controversa: alcuni autori hanno ottenuto risultati positivi altri no $(25,55)$.

Reddan et al (19) hanno condotto una rassegna sistematica della letteratura di trials prospettici, randomizzati, controllati, pubblicati tra il 2000 e il 2008, che paragonavano tra loro i MC e le strategie di prevenzione della Nefropatia da MC. La maggior parte dei trials paragonava $\mathrm{N}$-Acetilcisteina vs non N-Acetilcisteina: 6 trials hanno dimostrato un effetto protettivo significativo, uno un effetto benefico borderline e uno un effetto nocivo; ben 15 non hanno mostrato alcuna differenza nell'incidenza della Nefropatia tra trattamento con $\mathrm{N}$-Acetilcisteina e non trattamento $(56,57)$.

È stato dimostrato che il pretrattamento con N-Acetilcisteina riduce la citotossicità dei $\mathrm{MC}$ sulle cellule embrionali renali umane trattate con ioxitalamato, iopromide e iodixanolo (58) e migliora il danno renale acuto in modelli animali (59). II dosaggio suggerito è di $600 \mathrm{mg}$ per via orale due volte al giorno (il giorno prima e il giorno dell'esame radiologico); in alternativa una dose e.v. di $150 \mathrm{mg} / \mathrm{kg}$ mezz'ora prima del mezzo di contrasto oppure $50 \mathrm{mg} / \mathrm{kg}$ somministrato nell'arco di 4 ore.

Un altro antiossidante usato è l'Acido Ascorbico (3 g per os 2 ore prima e $2 \mathrm{~g}$ nella notte e al mattino successivo all'esame radiologico) (Fig. 2) $(39,60)$. I risultati ottenuti da vari autori sono controversi. Tuttavia, in una recente meta-analisi che coinvolgeva 1536 pazienti l'Acido Ascorbico è risultato aver ridotto del 33\% il rischio di Nefropatia da mezzo di con- trasto (61).

In un confronto tra N-Acetilcisteina e Acido Ascorbico, la N-Acetilcisteina (alla dose di $1200 \mathrm{mg}$ per os due volte al giorno nel giorno precedente e nel giorno della cateterizzazione coronarica) si è mostrata essere più efficace dell'Acido Ascorbico nel prevenire la Nefropatia da mezzo di contrasto, soprattutto nei diabetici con insufficienza renale cronica (62).

Un altro antiossidante, la Vitamina $E$, $(350 \mathrm{mg} / \mathrm{die} \mathrm{di}$ $\alpha$-tocoferolo o $300 \mathrm{mg} /$ die di $\nu$-tocoferolo 5 giorni prima di un intervento coronarico con iopromide e continuata per due giorni dopo l'intervento) in pazienti con insufficienza renale cronica riduceva l'incidenza della Nefropatia da MC dal 14.9\% del gruppo placebo al $4.9 \%$ con $\alpha$-tocoferolo e al $5.9 \%$ con $\gamma$-tocoferolo (63).

Un $\beta$-bloccante di terza generazione, il Nebivololo, è stato utilizzato ( $5 \mathrm{mg} /$ die per una settimana) per il suo effetto antiossidante e per l'azione vasodilatante attraverso un aumento del NO, con risultati incoraggianti (Fig. 2) (64-66).

Interessanti sono gli studi sugli effetti delle Statine nel prevenire la Nefropatia da MC (Fig. 2) (67-74). L'effetto nefroprotettivo delle Statine è stato attribuito non solo alle loro proprietà antiossidante, anti-infiammatoria e antitrombotica, ma anche alla loro attività vasodilatante mediante stimolazione del NO $(72,73)$.

La Simvastatina ha dimostrato un effetto vasoprotettivo dose-dipendente in animali sperimentali trattati con MC (72). Pazienti trattati con Pravastatina hanno mostrato tuttavia una minore incidenza della Nefropatia da MC rispetto ai pazienti 
trattati con Simvastatina $(75,76)$.

La Rosuvastatina $(10 \mathrm{mg} / \mathrm{die}$ per 5 giorni, iniziando 2 giorni prima della somministrazione del mezzo di contrasto) ha ridotto il rischio di Nefropatia da MC in pazienti con diabete e insufficienza renale cronica sottoposti ad arteriografia coronarica o periferica (77).

L'Atorvastatina può essere utilizzata per prevenire la Nefropatia da MC con i seguenti dosaggi: $40 \mathrm{mg} / \mathrm{die}$ per 3 giorni prima della angiografia coronarica (78); oppure $80 \mathrm{mg} 12$ ore prima dell' intervento coronarico percutaneo, più $40 \mathrm{mg}$ subito prima dell'intervento seguiti da un trattamento con $40 \mathrm{mg} /$ die (79).

\section{Citotossicità dei $M C$}

I MC, inoltre, hanno anche effetti citotossici diretti sia nei riguardi delle cellule endoteliali, che nei riguardi dell'epitelio tubulare renale, che portano alla apoptosi e a necrosi cellulare (Fig. 2).

Le cellule endoteliali sono le prime a giungere a contatto con il mezzo di contrasto in seguito alla sua iniezione intravascolare. II danno endoteliale, osservato al microscopio elettronico a scansione, consiste in protrusione nucleare, raggrinzamento cellulare, fenestrazione dello strato endoteliale e formazione di microvilli (blebbing) sulla membrana cellulare sino all'apoptosi (80). Pertanto la riduzione del NO midollare è dovuto non solo all'aumentata produzione delle ROS, ma anche alle cellule endoteliali danneggiate o apoptotiche (Fig. 2) (48). Le cellule endoteliali danneggiate possono anche liberare Endotelina che causa vasocostrizione (Fig. 2) (81).

Il mezzo di contrasto, dopo la somministrazione e.v. o intra-arteriosa, viene filtrato dai capillari glomerulari e raggiunge il lume dei tubuli renali prossimali dove viene marcatamente concentrato in virtù del notevole riassorbimento idrosalino da parte dei tubuli prossimali; la sua elevata concentrazione rende più elevata la sua citotossicità. $\mathrm{Ne}$ fa così le spese l'epitelio tubulare che viene gravemente danneggiato sino all'apoptosi. Questa azione citotossica diretta è stata dimostrata in vitro in segmenti tubulari isolati messi a contatto con il mezzo di contrasto $(82,83)$.

Lo studio delle alterazioni biochimiche, che sono alla base del danno dell'epitelio tubulare causato dal mezzo di contrasto, sono stati estesi alla ricerca, in cellule tubulari renali coltivate in vitro (84), delle alterazioni delle principali vie dei segnali intracellulari coinvolte nella sopravvivenza cellulare, nella morte delle cellule e nell'infiammazione (46, 85-92). Studi recenti su cellule tubulari primarie umane e su cellule HK-2 esposte a differenti MC hanno chiarito questi aspetti. Andreucci et al (89) hanno dimostrato una diminuita sopravvivenza cellulare secondaria a una ridotta attivazione di Akt/ PKB e di ETK 1/2, chinasi note di svolgere un ruolo pilota nella sopravvivenza/proliferazione cellulari, che veniva ridotta dalla transfezione delle cellule HK-2 con una attiva forma di Akt/PKB. Gli stessi autori hanno dimostrato, in cellule HK-2, che i MC agiscono sulla attivazione/disattivazione dei fattori di trascrizione, come Fox03a e STAT3, che controllano i geni interessati all'apoptosi e alla proliferazione cellulare $(87,90)$. Studi su animali, sia in vivo che in vitro, suggeriscono che i $\mathrm{MC}$ iodati possono causare direttamente un'apoptosi, mediata dalle caspasi, delle cellule tubulari renali (93). L'apoptosi indotta dai MC può essere dovuta alla attivazione di proteine shock e alla concorrente inibizione di enzimi citoprotettivi e prostaglandine $(94,95)$. Questo tipo di ricerche sperimentali può consentire di trovare i mezzi per contrastare la citotossicità dei MC sull'epitelio tubulare (96).

Pertanto il mezzo di contrasto causa una caduta del GFR: 1) per una vasocostrizione renale che causa una diminuzione del RBF; 2) per un danno epiteliale tubulare da tossicità diretta del mezzo di contrasto; 3) per una diuresi osmotica che causa ostruzione tubulare, con punti cruciali rappresentati dall'ipossia midollare e dallo stress ossidativo (Fig. 2).

\section{Disclosures}

Financial support: No financial support was received for this submission.

Conflict of interest: The author has no conflict of interest.

\section{Bibliografia}

1. Mehta RL, Kellum JA, Shah SV, et al. Acute Kidney Injury Network. Acute Kidney Injury Network: report of an initiative to improve outcomes in acute kidney injury. Crit Care. 2007; 11(2):R31.

2. Dirkes S. Acute kidney injury: not just acute renal failure anymore? Crit Care Nurse. 2011;31(1):37-49.

3. Schrier RW. ARF, AKI, or ATN? Nat Reviews Nephrol. 2010; 6(3):125.

4. Lattanzio MR, Kopyt NP. Acute kidney injury: new concepts in definition, diagnosis, pathophysiology, and treatment. J Am Osteopath Assoc. 2009;109(1):13-9.

5. Andreucci M. Side effects of radiographic contrast media. Biomed Res Int. 2014;2014:872574.

6. Andreucci M, Solomon R, Tasanarong A. Side effects of radiographic contrast media: pathogenesis, risk factors, and prevention. Biomed Res Int. 2014;2014:741018.

7. Andreucci M. [Radiographic contrast nephropathy]. G Ital Nefrol. 2014;31(5).

8. Sendeski M, Patzak A, Pallone TL, Cao C, Persson AE, Persson PB. lodixanol, constriction of medullary descending vasa recta, and risk for contrast medium-induced nephropathy. Radiology. 2009;251(3):697-704.

9. Arakawa $\mathrm{K}$, Suzuki $\mathrm{H}$, Naitoh $\mathrm{M}$, et al. Role of adenosine in the renal responses to contrast medium. Kidney Int. 1996; 49(5):1199-206.

10. Andreucci M, Faga T, Pisani A, Sabbatini M, Michael A. Pathogenesis of acute renal failure induced by iodinated radiographic contrast media. Austin J Nephrol Hypertens. 2014;1(1):1005.

11. Heyman SN, Rosen S, Rosenberger C. Renal parenchymal hypoxia, hypoxia adaptation, and the pathogenesis of radiocontrast nephropathy. Clin J Am Soc Nephrol. 2008;3(1):288-96.

12. Seeliger $E$, Flemming $B$, Wronski $T$, et al. Viscosity of contrast media perturbs renal hemodynamics. J Am Soc Nephrol. 2007; 18(11):2912-20.

13. Seeliger E, Lenhard DC, Persson PB. Contrast media viscosity versus osmolality in kidney injury: lessons from animal studies. Biomed Res Int. 2014;2014:358136. 
14. Hoste EA, De Waele JJ, Gevaert SA, Uchino S, Kellum JA. Sodium bicarbonate for prevention of contrast-induced acute kidney injury: a systematic review and meta-analysis. Nephrol Dial Transplant. 2010;25(3):747-58.

15. Jang JS, Jin HY, Seo JS, et al. Sodium bicarbonate therapy for the prevention of contrast-induced acute kidney injury - a systematic review and meta-analysis. Circ J. 2012;76(9):2255-65.

16. Masuda M, Yamada T, Mine T, et al. Comparison of usefulness of sodium bicarbonate versus sodium chloride to prevent contrast-induced nephropathy in patients undergoing an emergent coronary procedure. Am J Cardiol. 2007;1 00(5):781-6.

17. Navaneethan SD, Singh S, Appasamy S, Wing RE, Sehgal AR. Sodium bicarbonate therapy for prevention of contrast-induced nephropathy: a systematic review and meta-analysis. Am J Kidney Dis. 2009;53(4):617-27.

18. Tamura A, Goto Y, Miyamoto K, et al. Efficacy of single-bolus administration of sodium bicarbonate to prevent contrastinduced nephropathy in patients with mild renal insufficiency undergoing an elective coronary procedure. Am J Cardiol. 2009;104(7):921-5.

19. Reddan D, Laville M, Garovic VD. Contrast-induced nephropathy and its prevention: What do we really know from evidencebased findings? J Nephrol. 2009;22(3):333-51.

20. Zoungas S, Ninomiya T, Huxley R, et al. Systematic review: sodium bicarbonate treatment regimens for the prevention of contrast-induced nephropathy. Ann Intern Med. 2009;151(9): 631-8.

21. Brar SS, Shen AY, Jorgensen MB, et al. Sodium bicarbonate vs sodium chloride for the prevention of contrast mediuminduced nephropathy in patients undergoing coronary angiography: a randomized trial. JAMA. 2008;300(9):1038-46.

22. Brar SS, Hiremath S, Dangas G, Mehran R, Brar SK, Leon MB. Sodium bicarbonate for the prevention of contrast inducedacute kidney injury: a systematic review and meta-analysis. Clin J Am Soc Nephrol. 2009;4(10):1584-92.

23. Shavit L, Korenfeld R, Lifschitz M, Butnaru A, Slotki I. Sodium bicarbonate versus sodium chloride and oral $\mathrm{N}$-acetylcysteine for the prevention of contrast-induced nephropathy in advanced chronic kidney disease. J Interv Cardiol. 2009;22(6):556-63.

24. Vasheghani-Farahani A, Sadigh G, Kassaian SE, et al. Sodium bicarbonate plus isotonic saline versus saline for prevention of contrast-induced nephropathy in patients undergoing coronary angiography: a randomized controlled trial. Am J Kidney Dis. 2009;54(4):610-8.

25. From AM, Bartholmai BJ, Williams AW, Cha SS, Pflueger A, McDonald FS. Sodium bicarbonate is associated with an increased incidence of contrast nephropathy: a retrospective cohort study of 7977 patients at mayo clinic. Clin J Am Soc Nephrol. 2008;3(1):10-8

26. Ad-hoc working group of ERPB, Fliser D, Laville M, et al. A European Renal Best Practice (ERBP) position statement on the Kidney Disease Improving Global Outcomes (KDIGO) clinical practice guidelines on acute kidney injury: part 1: definitions, conservative management and contrast-induced nephropathy. Nephrol Dial Transplant. 2012;27(12):4263-72.

27. Erley CM, Duda SH, Rehfuss $D$, et al. Prevention of radiocontrast-media-induced nephropathy in patients with pre-existing renal insufficiency by hydration in combination with the adenosine antagonist theophylline. Nephrol Dial Transplant. 1999;14(5):1146-9.

28. Huber W, Ilgmann K, Page $M$, et al. Effect of theophylline on contrast material-nephropathy in patients with chronic renal insufficiency: controlled, randomized, double-blinded study. Radiology. 2002;223(3):772-9.

29. Shammas NW, Kapalis MJ, Harris M, McKinney D, Coyne EP.
Aminophylline does not protect against radiocontrast nephropathy in patients undergoing percutaneous angiographic procedures. J Invasive Cardiol. 2001;13(11):738-40.

30. Toprak O. Conflicting and new risk factors for contrast induced nephropathy. J Urol. 2007;178(6):2277-83.

31. Kalyesubula R, Bagasha P, Perazella MA. ACE-I/ARB therapy prior to contrast exposure: what should the clinician do? Biomed Res Int. 2014;2014:423848.

32. Rim MY, Ro H, Kang WC, et al. The effect of renin-angiotensinaldosterone system blockade on contrast-induced acute kidney injury: a propensity-matched study. Am J Kidney Dis. 2012;60(4):576-82.

33. Umruddin Z, Moe K, Superdock K. ACE inhibitor or angiotensin II receptor blocker use is a risk factor for contrast-induced nephropathy. J Nephrol. 2012;25(5):776-81.

34. Onuigbo MA, Onuigbo NT. Does renin-angiotensin aldosterone system blockade exacerbate contrast-induced nephropathy in patients with chronic kidney disease? A prospective 50-month Mayo Clinic study. Ren Fail. 2008;30(1):67-72.

35. Rosenstock JL, Bruno R, Kim JK, et al. The effect of withdrawal of ACE inhibitors or angiotensin receptor blockers prior to coronary angiography on the incidence of contrast-induced nephropathy. Int Urol Nephrol. 2008, 40(3):749-55.

36. Group KDIGOKAKI. KDIGO Clinical Practice Guideline for Acute Kidney Injury. Kidney international. 2012;2(Suppl):S1-138.

37. Koch JA, Plum J, Grabensee B, Modder U. Prostaglandin E1: a new agent for the prevention of renal dysfunction in high risk patients caused by radiocontrast media? PGE1 Study Group. Nephrol Dial Transplant. 2000;15(1):43-9.

38. Sketch MH Jr, Whelton A, Schollmayer E, et al; Prostaglandin E1 Study Group. Prevention of contrast media-induced renal dysfunction with prostaglandin E1: a randomized, double-blind, placebo-controlled study. Am J Ther. 2001;8(3):155-62.

39. Spargias K, Alexopoulos E, Kyrzopoulos S, et al. Ascorbic acid prevents contrast-mediated nephropathy in patients with renal dysfunction undergoing coronary angiography or intervention. Circulation. 2004;110(18):2837-42.

40. Spargias K, Adreanides E, Demerouti E, et al. lloprost prevents contrast-induced nephropathy in patients with renal dysfunction undergoing coronary angiography or intervention. Circulation. 2009;120(18):1793-9.

41. Neyra JA, Shah S, Mooney R, Jacobsen G, Yee J, Novak JE. Contrast-induced acute kidney injury following coronary angiography: a cohort study of hospitalized patients with or without chronic kidney disease. Nephrol Dial Transplant. 2013; 28(6):1463-71.

42. Briguori C, Airoldi F, Visconti G, et al. Novel approaches for preventing or limiting events in diabetic patients (Naples-diabetes) trial: a randomized comparison of 3 drug-eluting stents in diabetic patients. Circ Cardiovasc Interv. 2011;4(2):121-9.

43. Briguori C, Visconti G, Focaccio A, et al; REMEDIAL II Investigators. Renal Insufficiency After Contrast Media Administration Trial II (REMEDIAL II): RenalGuard System in high-risk patients for contrast-induced acute kidney injury. Circulation. 2011;124(11):1260-9.

44. Briguori C. Renalguard system: a dedicated device to prevent contrast-induced acute kidney injury. Int J Cardiol. 2013; 168(2):643-4.

45. Marenzi G, Ferrari C, Marana I, et al. Prevention of contrast nephropathy by furosemide with matched hydration: the MYTHOS (Induced Diuresis with Matched Hydration Compared to Standard Hydration for Contrast Induced Nephropathy Prevention) trial. JACC Cardiovasc Interv. 2012;5(1):90-7.

46. Sabbatini $M$, Santillo $M$, Pisani $A$, et al. Inhibition of Ras/ ERK1/2 signaling protects against postischemic renal injury. 
Am J Physiol Renal Physiol. 2006;290(6):F1408-15.

47. Heyman SN, Rosen S, Khamaisi M, Idee JM, Rosenberger C. Reactive oxygen species and the pathogenesis of radiocontrastinduced nephropathy. Invest Radiol. 2010;45(4):188-95.

48. Sendeski MM. Pathophysiology of renal tissue damage by iodinated contrast media. Clin Exp Pharmacol Physiol. 2011; 38(5):292-9.

49. Pisani A, Riccio $E$, Andreucci M, et al. Role of reactive oxygen species in pathogenesis of radiocontrast-induced nephropathy. Biomed Res Int. 2013;2013:868321.

50. Pacher P, Beckman JS, Liaudet L. Nitric oxide and peroxynitrite in health and disease. Physiol Rev. 2007;87(1):315-24.

51. Myers SI, Wang L, Liu F, Bartula LL. lodinated contrast induced renal vasoconstriction is due in part to the downregulation of renal cortical and medullary nitric oxide synthesis. J Vasc Surg. 2006;44(2):383-91.

52. Pisani A, Sabbatini M, Riccio E, et al. Effect of a recombinant manganese superoxide dismutase on prevention of contrast-induced acute kidney injury. Clin Exp Nephrol. 2014;18(3):424-31.

53. Sardella G, Briguori C, Garbo R, et al. Evidence from the Resorbable-polymer stent versus Unresorbable-polymer stent Deployment for coronary Intervention: (RUDI-2) registry. Int J Cardiol. 2014;15;172(2):472-5.

54. Safirstein R, Andrade L, Vieira JM. Acetylcysteine and nephrotoxic effects of radiographic contrast agents-a new use for an old drug. N Engl J Med. 2000;343(3):210-2.

55. Gurm HS, Smith DE, Berwanger O, et al; BMC2 (Blue Cross Blue Shield of Michigan Cardiovascular Consortium). Contemporary use and effectiveness of $\mathrm{N}$-acetylcysteine in preventing contrast-induced nephropathy among patients undergoing percutaneous coronary intervention. JACC Cardiovasc Interv. 2012;5(1):98-104.

56. Coyle LC, Rodriguez A, Jeschke RE, Simon-Lee A, Abbott KC, Taylor AJ. Acetylcysteine In Diabetes (AID): a randomized study of acetylcysteine for the prevention of contrast nephropathy in diabetics. Am Heart J. 2006;151(5):1032 e9-12.

57. Sandhu C, Belli AM, Oliveira DB. The role of $\mathrm{N}$-acetylcysteine in the prevention of contrast-induced nephrotoxicity. Cardiovasc Intervent Radiol. 2006;29(3):344-7.

58. Lee HC, Sheu SH, Liu IH, et al. Impact of short-duration administration of $\mathrm{N}$-acetylcysteine, probucol and ascorbic acid on contrast-induced cytotoxicity. J Nephrol. 2012;25(1):56-62.

59. DiMari J, Megyesi J, Udvarhelyi N, Price P, Davis R, Safirstein R. $\mathrm{N}$-acetyl cysteine ameliorates ischemic renal failure. Am J Physiol. 1997;272(3 Pt 2):F292-8.

60. Alexopoulos E, Spargias K, Kyrzopoulos S, et al. Contrastinduced acute kidney injury in patients with renal dysfunction undergoing a coronary procedure and receiving non-ionic low-osmolar versus iso-osmolar contrast media. Am J Med Sci. 2010;339(1):25-30.

61. Sadat U, Walsh SR, Norden AG, Gillard JH, Boyle JR. Does oral $\mathrm{N}$-acetylcysteine reduce contrast-induced renal injury in patients with peripheral arterial disease undergoing peripheral angiography? A randomized-controlled study. Angiology. 2011;62(3):225-30.

62. Jo SH, Koo BK, Park JS, et al. N-acetylcysteine versus Ascorbic acid for preventing contrast-Induced nephropathy in patients with renal insufficiency undergoing coronary angiography NASPI study-a prospective randomized controlled trial. Am Heart J. 2009;157(3):576-83.

63. Tasanarong A, Vohakiat A, Hutayanon P, Piyayotai D. New strategy of alpha- and gamma-tocopherol to prevent contrastinduced acute kidney injury in chronic kidney disease patients undergoing elective coronary procedures. Nephrol Dial Transplant. 2013;28(2):337-44.
64. Toprak O, Cirit M, Tanrisev M, et al. Preventive effect of nebivolol on contrast-induced nephropathy in rats. Nephrol Dial Transplant. 2008;23(3):853-9.

65. Gunebakmaz O, Kaya MG, Koc F, et al. Does nebivolol prevent contrast-induced nephropathy in humans? Clin Cardiol. 2012;35(4):250-4.

66. Avci E, Yesil M, Bayata S, Postaci N, Arikan E, Cirit M. The role of nebivolol in the prevention of contrast-induced nephropathy in patients with renal dysfunction. Anadolu Kardiyol Derg. 2011;11(7):613-7.

67. Khanal S, Attallah N, Smith DE, et al. Statin therapy reduces contrast-induced nephropathy: an analysis of contemporary percutaneous interventions. Am J Med. 2005;118(8):843-9.

68. Patti G, Nusca A, Chello M, et al. Usefulness of statin pretreatment to prevent contrast-induced nephropathy and to improve long-term outcome in patients undergoing percutaneous coronary intervention. Am J Cardiol. 2008;101(3):279-85.

69. Leoncini M, Toso A, Maioli M, Tropeano F, Bellandi F. Statin treatment before percutaneous coronary intervention. J Thorac Dis. 2013;5(3):335-42.

70. Zhang BC, Li WM, Xu YW. High-dose statin pretreatment for the prevention of contrast-induced nephropathy: a meta-analysis. Can J Cardiol. 2011;27(6):851-8.

71. Andreucci M. [Statins in CIN: a problem at least partly solved?]. G Ital Nefrol. 2013;30(3).

72. Al-Otaibi KE, Al Elaiwi AM, Tariq M, Al-Asmari AK. Simvastatin attenuates contrast-induced nephropathy through modulation of oxidative stress, proinflammatory myeloperoxidase, and nitric oxide. Oxid Med Cell Longev. 2012;2012: 831748.

73. Quintavalle C, Fiore D, De Micco F, et al. Impact of a high loading dose of atorvastatin on contrast-induced acute kidney injury. Circulation. 2012;126(25):3008-16.

74. Sabbatini M, Pisani A, Uccello F, et al. Atorvastatin improves the course of ischemic acute renal failure in aging rats. J Am Soc Nephrol. 2004;15(4):901-09.

75. Munoz MA, Maxwell PR, Green K, Hughes DW, Talbert RL. Pravastatin versus simvastatin for prevention of contrastinduced nephropathy. J Cardiovasc Pharmacol Ther. 2011;16 (3-4):376-9.

76. Yoshida S, Kamihata H, Nakamura S, et al. Prevention of contrast-induced nephropathy by chronic pravastatin treatment in patients with cardiovascular disease and renal insufficiency. J Cardiol. 2009, 54(2):192-8.

77. Han Y, Zhu G, Han L, et al. Short-term rosuvastatin therapy for prevention of contrast-induced acute kidney injury in patients with diabetes and chronic kidney disease. J Am Coll Cardiol. 2014;63(1):62-70.

78. Acikel S, Muderrisoglu H, Yildirir A, et al. Prevention of contrast-induced impairment of renal function by short-term or long-term statin therapy in patients undergoing elective coronary angiography. Blood Coagul Fibrinolysis. 2010; 21(8):750-7.

79. Patti G, Ricottini E, Nusca A, et al. Short-term, high-dose Atorvastatin pretreatment to prevent contrast-induced nephropathy in patients with acute coronary syndromes undergoing percutaneous coronary intervention (from the ARMYDA-CIN [atorvastatin for reduction of myocardial damage during angioplasty-contrast-induced nephropathy] trial. Am J Cardiol. 2011;108(1):1-7.

80. Gospos C, Freudenberg N, Staubesand J, Mathias K, Papacharlampos $\mathrm{X}$. The effect of contrast media on the aortic endothelium of rats. Radiology. 1983;147(3):685-8.

81. Heyman SN, Clark BA, Kaiser N, et al. Radiocontrast agents induce endothelin release in vivo and in vitro. J Am Soc Nephrol. 
1992;3(1):58-65.

82. Hardiek K, Katholi RE, Ramkumar V, Deitrick C. Proximal tubule cell response to radiographic contrast media. Am J Physiol Renal Physiol. 2001;280(1):F61-70.

83. Heinrich MC, Kuhlmann MK, Grgic A, Heckmann M, Kramann B, Uder M. Cytotoxic effects of ionic high-osmolar, nonionic monomeric, and nonionic iso-osmolar dimeric iodinated contrast media on renal tubular cells in vitro. Radiology. 2005;235(3):843-9.

84. Andreucci M, Faga T, Lucisano G, et al. Mycophenolic acid inhibits the phosphorylation of NF-kappaB and JNKs and causes a decrease in IL-8 release in $\mathrm{H}_{2} \mathrm{O}_{2}$-treated human renal proximal tubular cells. Chem Biol Interact. 2010;185(3):253-62.

85. Andreucci M, Michael A, Kramers C, et al. Renal ischemia/ reperfusion and ATP depletion/repletion in LLC-PK(1) cells result in phosphorylation of FKHR and FKHRL1. Kidney Int. 2003;64(4):1189-98.

86. Andreucci M, Fuiano G, Presta P, et al. Downregulation of cell survival signalling pathways and increased cell damage in hydrogen peroxide-treated human renal proximal tubular cells by alpha-erythropoietin. Cell Prolif. 2009;42(4):554-61.

87. Andreucci M, Lucisano G, Faga T, et al. Differential activation of signaling pathways involved in cell death, survival and inflammation by radiocontrast media in human renal proximal tubular cells. Toxicol Sci. 2011;119(2):408-16.

88. Andreucci M. [Contrast media and nephrotoxicity: a molecular conundrum]. G Ital Nefrol. 2011;28(4):355.
89. Andreucci M, Fuiano G, Presta P, et al. Radiocontrast media cause dephosphorylation of Akt and downstream signaling targets in human renal proximal tubular cells. Biochem Pharmacol. 2006;72(10):1334-42.

90. Andreucci M, Faga T, Russo D, et al. Differential activation of signaling pathways by low-osmolar and iso-osmolar radiocontrast agents in human renal tubular cells. J Cell Biochem. 2014;115(2):281-9.

91. Quintavalle $C$, Brenca M, De Micco F, et al. In vivo and in vitro assessment of pathways involved in contrast media-induced renal cells apoptosis. Cell Death Dis. 2011;2:e155.

92. Michael A, Faga T, Pisani A, et al. Molecular mechanisms of renal cellular nephrotoxicity due to radiocontrast media. Biomed Res Int. 2014;2014:249810.

93. Lee HC, Chang JG, Yen HW, Liu IH, Lai WT, Sheu SH. Ionic contrast media induced more apoptosis in diabetic kidney than nonionic contrast media. J Nephrol. 2011;24(3):376-80.

94. Cunha MA, Schor N. Effects of gentamicin, lipopolysaccharide, and contrast media on immortalized proximal tubular cells. Ren Fail. 2002;24(6):687-90.

95. Peer A, Averbukh Z, Berman S, Modai D, Averbukh M, Weissgarten J. Contrast media augmented apoptosis of cultured renal mesangial, tubular, epithelial, endothelial, and hepatic cells. Invest Radiol. 2003;38(3):177-82.

96. Andreucci M, Faga T, Pisani A, et al. Reversal of radiocontrast medium toxicity in human renal proximal tubular cells by white grape juice extract. Chem Biol Interact. 2015;229:17-25. 